\title{
Relativistically invariant self-similarity approach for description of collective phenomena
}

\author{
E. G. Baldina ${ }^{1,2,}$ and A. A. Baldin ${ }^{1,2}$ \\ ${ }^{1}$ Joint Institute for Nuclear Research, Dubna, 141980 Russia \\ ${ }^{2}$ Institute for Advanced Studies “OMEGA”, Dubna, 141986 Russia
}

\begin{abstract}
The self-similarity approach is applied to description of nuclear interactions. The obtained self-similarity solution quantitatively describes particle production in relativistic nuclear collisions. It is an appropriate tool for planning and optimization of experiments aimed at the search of new collective phenomena in highly excited nuclear matter, especially in the intermediate energy range.
\end{abstract}

Self-similarity is a special symmetry of solutions when the change in scales of independent variables can be compensated by the self-similarity transformation of other dynamic variables. This results in the reduction of the number of variables in any physical law. Self-similarity is an important tool for investigation of complex physical phenomena with the objective of finding regularities of general character coupling the multitude of experimentally measurable data.

Historically, self-similarity in elementary particle physics was introduced in relation with the deep inelastic electron scattering on protons, it is the well known Bjorken scaling [1] obtained directly from the conservation laws in the assumption since the squared momentum of particles participating in the reaction is much greater than the squared mass, the latter can be neglected.

Writing the momentum conservation law of the reaction, we have

$$
P_{1}+x P_{2}=P_{1}^{\prime}+\sum P_{i}^{\prime}
$$

where the subscript "1" denotes the electron, the subscript " 2 " the proton, and prime marks the quantities attributed to the particles after the interaction.

Introducing the so called Bjorken variable, $P_{1}-P_{1}^{\prime}=q$ and making simple transformations of (1) with account of the fact that the cross-products in the sum describing relative motion of all other particles produced in the reaction can be neglected, and finally, neglecting the squared masses of particles, as compared to the squared three-momenta, we get the known Bjorken scaling in the form

$$
x=-\frac{q}{2 P_{2} q} .
$$

The general self-similar solution [2,3] including the Bjorken solution as a particular case, is obtained as follows.

\footnotetext{
* Corresponding author: e.baldina@mail.ru
} 
We consider two interacting nuclei and assume that fractions $X_{1}$ and $X_{2}$ of masses (or fractions of momenta) of these nuclei participate in the interaction. The 4-momentum conservation is written as:

$$
X_{1} P_{1}+X_{2} P_{2}=P_{1}^{\prime}+P_{2}^{\prime}+P_{3}+\sum P_{i}^{\prime}
$$

Here, $P_{3}$ is the 4-momentum of the particle of interest produced in the reaction (note that conservation of quantum numbers should be taken into account explicitly by addition of corresponding terms to the right-hand side of (3)).

Again, we neglect terms of relative motion of produced particles and masses of all particles not registered, and seek the self-similar solution in the form

$$
E \frac{d^{3} \sigma}{d p^{3}}=C_{1} A_{1}^{\alpha\left(X_{1}\right)} A_{2}^{\alpha\left(X_{2}\right)} f(\Pi)
$$

where $\Pi=\frac{1}{2}\left(X_{1}^{2}+X_{2}^{2}+2 X_{1} X_{2} \gamma_{12}\right)^{\frac{1}{2}}$ is the similarity parameter.

The fractions $X_{1}$ and $X_{2}$ are found by minimization of $\Pi$ (figure 1) (see also [4]).

$$
E \frac{d^{3} \sigma}{d p^{3}}=C_{1} A_{1}^{1 / 3\left(1+X_{1}\right)} A_{2}^{1 / 3\left(1+X_{2}\right)} \exp \left(-\Pi / C_{2}\right)
$$

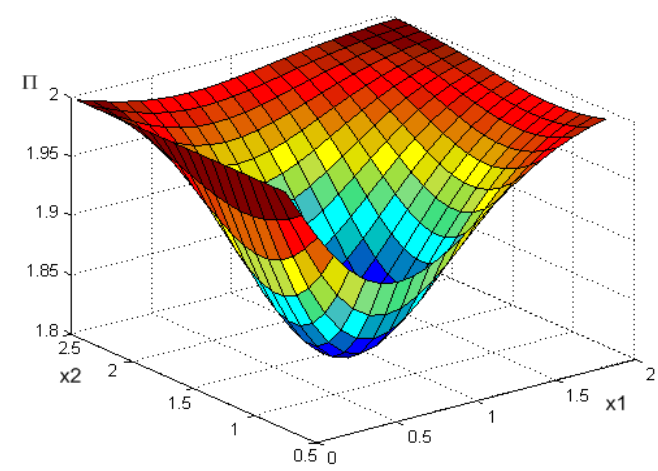

Figure 1. Minimum of $\Pi$ in terms of X1, X2 coordinates.

This self-similar solution describes quantitatively a wide variety of experimental data on cumulative, twice cumulative particle production, and antimatter production [5-7] (figure 2).

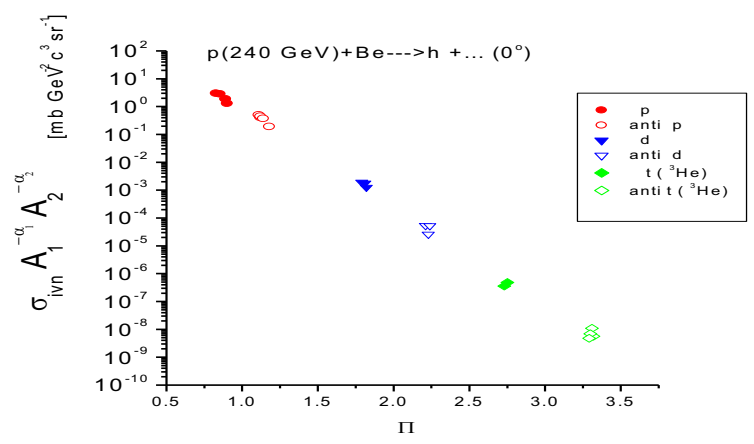

Figure 2. Matter and antimatter production: the self-similarity description. 
It should be underlined that the widely used assumption $P^{2}>M^{2}$ is not valid even for very high momenta. It will be shown below that even a small mass difference essentially influences the reaction mechanisms.

The above self-similarity solution was found for reactions with low and intermediate transverse momenta (below $1 \mathrm{GeV} / \mathrm{c}$ ). Recent experiment at the Institute for High Energy Physics (Protvino) [8], however, demonstrated that the A-dependence of the form $A^{\left(1+X_{2}\right) / 3}$ well describes the dynamic dependence of the cross sections on $X_{2}$, while it fails to describe a stronger dependence on the mass number of the nucleus observed in this experiment (figure 3).

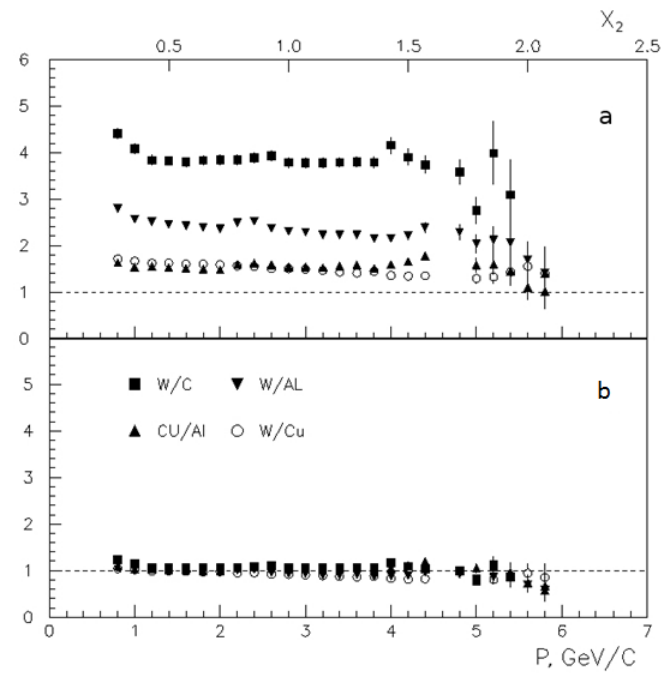

Figure 3. Ratio of cross sections of negative pion production on different nuclei multiplied by inverse A-dependence (see the text). The lower axis shows the momentum, the upper axis, $X_{2}$. (a) The ratios are obtained using the A-dependence in the form $A^{\left(1+X_{2}\right) / 3}$, (b) the ratios are obtained using the Adependence in the form $A^{\left(2.45+X_{2}\right) / 3}$ [8].

It is possible to develop the self-similarity solution for description of high- $P_{t}$ reactions; for this purpose the function $f(\Pi)$ in (4) should be added by a term depending on $P_{t}$.

The scientific program of research at the NICA collider [9], in the field of relativistic nuclear collision includes the study of the phenomenon of enhanced $\mathrm{K}^{+}$production in nucleon-nucleon collisions in the intermediate energy range, $\sqrt{S} \sim 3 \div 12 \mathrm{GeV}$ [10]. This phenomenon was observed in cumulative processes and is well described by the above selfsimilarity solution. The specific feature of this description is that it is necessary to take into account the channels with $\Lambda \Sigma^{0}, \Sigma^{+}$, and $\Sigma^{-}$hyperons for description of $\mathrm{K}$ meson production in the framework of self-similarity solution (4). Taking into account the contribution of all these channels yields the quantitative description of the enhanced $\mathrm{K}^{+}$ production. This is a persuasive illustration that small mass corrections cannot be neglected in relativistic particle production.

Collective nuclear effects in the intermediate energy range have received great attention recently. Experiments in this field have been performed at SPS (CERN), are planned at NICA collider (JINR), FAIR (GSI), and RHIC (BNL). These experiments are aimed at investigation of exotic collective properties of matter in heavy ion interactions. 
The self-similarity solution presented here well describes collective phenomena; therefore, it is reasonable to apply it for optimization of the future experiments at NICA aimed at the observation of these effects. Let us apply this self-similarity solution to predict the kinematic range where the particle production cross sections will be the largest or the most experimentally convenient.

Let us consider $\phi$ meson production in heavy ion collisions in the intermediate energy range.

Figure 4 shows the predicted invariant cross section of $\phi$ production and $\phi$ yield as a function of momentum in the laboratory reference frame in collision of $25 \mathrm{AGeV}$ gold ions (we consider fixed target for convenience). Obviously, the particle yield changes by orders of magnitude with varying angle, which is extremely important from the experimental point of view. It should also be noted that the cross section maximum shifts toward the central region with increasing angle of emitted particle.

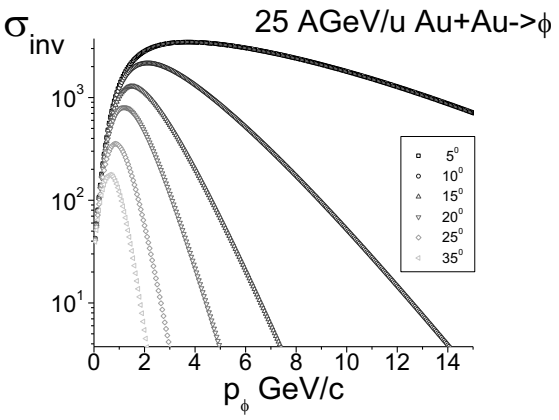

a)

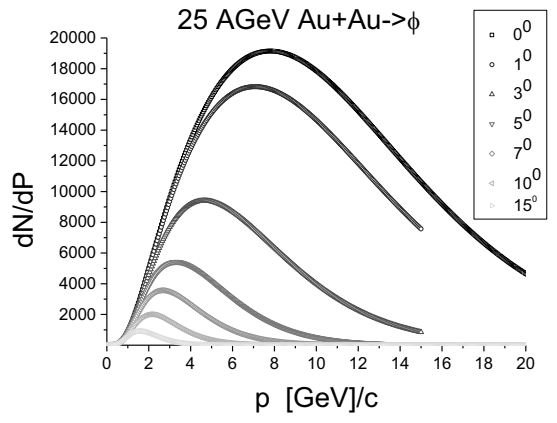

b)

Figure 4. (a) Predicted invariant cross section of $\phi$ production as a function of particle momentum for different angles; (b) $\phi$ meson yield as a function of momentum for different angles.

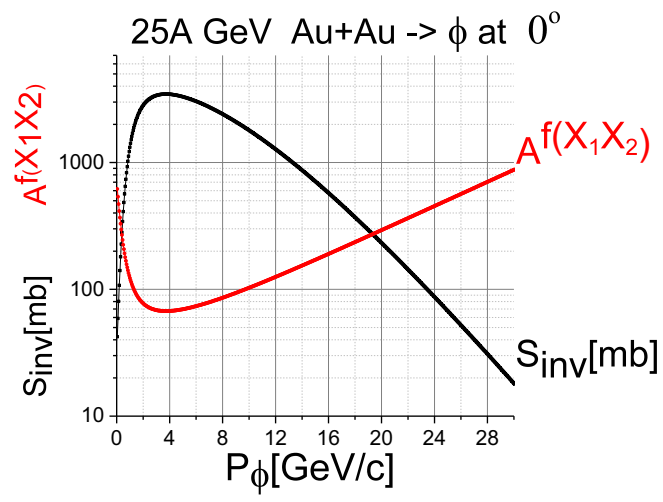

Figure 5. Invariant cross section and A-dependence of $\phi$ meson production in gold-gold collision at $25 \mathrm{AGeV}$ and zero angle.

Figure 5 demonstrates that the maximum cross section corresponds to the minimum Adependence, which means minimum manifestation of collective phenomena. This is especially important for experiments in which the ratios of particle yields are studied. 
Therefore, it is extremely important to take into account the acceptance of the particular experimental setup in data analysis of such experiments.

In conclusion, we state the following. The developed functional self-similarity solution quantitatively describes angular, energy and A-dependences of inclusive production cross sections of all hadrons with transverse momentum up to $1 \mathrm{GeV} / \mathrm{c}$. Higher transverse momenta require an additional mechanism to account for momentum dependence in the invariant cross section.

The analysis of inclusive spectra for the data selected in different ways shows that multiplicity in relativistic nuclear collisions has its origin basically in independent nucleonnucleon interactions.

Interaction of very heavy nuclei ( $\mathrm{Au}, \mathrm{Pb}$, etc.) may "entangle" the experimental picture of the reaction and complicate theoretical interpretation. Collective effects can be observed already for light and intermediate nuclei, although with lower multiplicity and combinatorial background.

Collective phenomena become extinct with increasing collision energy. Therefore, the energy range from hundreds $\mathrm{MeV}$ to tens of $\mathrm{GeV}$ is optimal for experimental observation of collective effects in nuclear collisions. Of interest is the study of the whole energy range available at the NICA collider, toward the lowest possible energies.

\section{References}

1. J. D. Bjorken, Phys. Rev. 179, 1547 (1969)

2. A. A. Baldin, Phys. Part. Nucl. 56(3), 385 (1993)

3. A. M. Baldin and A. A. Baldin, Phys. Part. Nucl. 29(3), 577 (1998)

4. V. S. Stavinskii, in Proc. of IX International Seminar on Problems of High Energy Physics 1, 190 (JINR, Dubna, 1988)

5. A. A. Baldin, E.G. Baldina, in Selected Papers of the Seminar (2000-2005) Symmerties and Integrable Systems, Ed. A. N. Sissakian, 1, 41 (JINR, Dubna, 2006)

6. A. A. Baldin et al., JINR Rapid Comm. 3(92), 20 (1992)

7. A.A. Baldin, E.G. Baldina, E.N. Kladnitskaya, and O.V. Rogachevsky, Phys. Part. Nucl. Lett. 121, 7 (2004)

8. V. V. Ammosov, et al., Phys. At. Nucl. 76, 1213 (2013)

9. V. Kekelidze, R. Lednicky, V. Matveev, I. Meshkov, A. Sorin, and G. Trubnikov, Phys. Part. Nucl. Lett. 9(4), 313 (2012)

10. C. Blume, J. Phys. G 31, S57 (2005) 